\title{
MEASURABLE SUBSPACES AND SUBALGEBRAS ${ }^{1}$
}

\section{R. R. BAHADUR}

1. Introduction. Let $(X, S, \mu)$ be a probability measure space. Here $X$ is a set of points $x, S$ is a $\sigma$-algebra of subsets of $X$, and $\mu$ is a $\sigma$-additive measure on $S$ with $\mu(X)=1$. Let $V=\mathcal{L}_{2}(X, S, \mu)$ be the real Hilbert space of $S$-measurable functions $f(x)$ with $\int_{X} f^{2} d \mu<\infty$.

For $f$ and $g$ in $V$, we write $(f, g)=\int_{X} f \cdot g d \mu$ where $(f \cdot g)(x) \equiv f(x) \cdot g(x)$, and $\|f\|=(f, f)^{1 / 2}$. Convergence in $V$ is defined as usual in the norm topology, that is to say, $\lim _{n \rightarrow \infty} f_{n}=f$ means $\lim _{n \rightarrow \infty}\left\|f_{n}-f\right\|=0$. If $f$ and $g$ are functions in $V$ such that $\|f-g\|=0$, they are regarded as identical and we write $f=g . f \geqq g$ means that $\mu\{x: f(x)<g(x)\}=0$. For any real $\alpha$, the function which is equal to $\alpha$ for every $x$ is also denoted by $\alpha$. A function $f$ in $V$ is said to be bounded if there exist $\alpha$ and $\beta$ such that $\alpha \leqq f \leqq \beta$.

A function on $X$ which takes only the values 0 and 1 is called a characteristic function. For any set $A \subset X, \chi_{A}$ denotes the characteristic function which equals 1 on $A$ and vanishes on $X-A$. If $S_{1}$ and $S_{2}$ are subclasses of $S$, we write $S_{1} \subset S_{2}$ if corresponding to each set $A \in S_{1}$ there exists a $B \in S_{2}$ such that $\chi_{B}=\chi_{A}$; we write $S_{1}=S_{2}$ if $S_{1} \subset S_{2}$ and $S_{2} \subset S_{1}$.

Let $W$ be a subspace ( $\equiv$ closed linear manifold) in $V . W$ is algebraic if it contains the function 1 (and therefore every constant function) and if, for any two bounded functions $f$ and $g$ in $W$, the function $f \cdot g$ is also in $W . W$ is bounded (so to speak) if the set of bounded functions in $W$ is dense in $W . W$ is measurable if there exists a (necessarily unique) $\sigma$-subalgebra of $S, S^{*}$ say, such that $W=\mathcal{L}_{2}\left(X, S^{*}, \mu\right)$, that is to say, $W$ is the set of all $S^{*}$-measurable functions in $V$.

Let $T$ be the (orthogonal) projection to the subspace $W . T$ is constant-preserving if $T \alpha=\alpha$ for every constant function $\alpha . T$ is positive if $f \geqq 0$ implies $T f \geqq 0$.

Let $S^{0}$ be the smallest $\sigma$-algebra of sets of $X$ such that each $f$ in $W$ is an $S^{0}$-measurable function. Let $S_{0}$ be the class of all sets $A \subset X$ such that $\chi_{A}$ is in $W$. While $S_{0}$ is not necessarily a $\sigma$-algebra, it is a nonempty class of sets, with $S_{0} \subset S^{0} \subset S$.

The main result of this note can then be stated as follows.

THEOREM. The following propositions are equivalent:

Received by the editors November 18, 1954.

1 This work was supported in part by the Office of Naval Research under contract N6onr-271, T.O. XI. 
(i) $T$ is constant-preserving and positive.

(ii) $W$ is algebraic and bounded.

(iii) $S_{0}=S^{0}$.

(iv) $W=\mathcal{L}_{2}\left(X, S^{0}, \mu\right)$.

The proof of the theorem is given in the next section.

By the equivalence of (ii) and (iv) we have

Corollary 1. A subspace is algebraic and bounded if and only if it is measurable.

This result is essentially about the set $\mathcal{L}_{\infty}$ of bounded functions in $V$, and it may be worthwhile to state it entirely in terms of $\mathcal{L}_{\infty}$ as follows. Regard $\mathcal{L}_{\infty}$ as a linear algebra under pointwise multiplication of functions, and for the moment suppose closure in $\mathcal{L}_{\infty}$ to mean closure in the usual $\mathcal{L}_{\infty}$ topology. Then Corollary 1 can be shown to be equivalent to the proposition that a closed linear subalgebra of $\mathcal{L}_{\infty}$ contains 1 if and only if it is a measurable algebra, that is to say, it is the closure of the linear algebra generated by a set of characteristic functions including 1 .

It is easy to show (cf. the last paragraph of the following section) that if $S^{*}$ is a $\sigma$-subalgebra of $S$ then the projection to $\mathcal{L}_{2}\left(X, S^{*}, \mu\right)$ is exactly the conditional expectation operator relative to $S^{*}$. In other words, a transformation $T$ is a conditional expectation if and only if $T$ is the projection to a measurable subspace. ${ }^{2}$ In consequence, the equivalence of (i) and (iv) yields the following characterization of conditional expectation.

Corollary 2. A transformation $T$ on $V$ into itself is a conditional expectation if and only if $T$ is linear, idempotent, self-adjoint, constantpreserving, and positive.

This result is closely related to a characterization of conditional expectation operators on $\mathcal{L}_{1}$ which was obtained by Moy [1, Theorem 2.2 ]. An essential part of the proof which follows is based on an argument of Moy.

2. Proof of the theorem. Since $T$ is a projection, we have

$$
\begin{aligned}
T(\alpha f+\beta g) & =\alpha T(f)+\beta T(g), \\
T^{2} f & =T f,
\end{aligned}
$$

and

$$
(T f, g)=(f, T g)
$$

2 This fact, which motivated the work presented here, was pointed out to the writer by L. J. Savage. 
for all $\alpha, \beta, f$ and $g$; moreover, since $T$ is the projection to $W$,

$$
W=\{T f: f \in V\},
$$

and also

$$
W=\{f: T f=f, f \in V\} .
$$

(Cf., e.g., [2].)

We shall prove the theorem by showing that (i) $\rightarrow$ (ii) $\rightarrow$ (iii) $\rightarrow$ (iv) $\rightarrow$ (i).

Suppose then that (i) holds, that is,

$$
T \alpha=\alpha
$$

for every $\alpha$

and

$$
T f \geqq 0 \quad \text { whenever } f \geqq 0 .
$$

It follows from (1) that (6) and (7) are equivalent to

$$
\alpha \leqq T f \leqq \beta \quad \text { whenever } \alpha \leqq f \leqq \beta
$$

and

$$
T f \geqq T g \quad \text { whenever } f \geqq g \text {. }
$$

Consider a fixed $g$ in $W$. Define $f_{n}(x)=g(x)$ if $|g(x)| \leqq n$ and $=0$ otherwise, for $n=1,2, \cdots$. Clearly, $\left\{f_{n}\right\}$ is a sequence in $V$ such that $\lim _{n \rightarrow \infty} f_{n}=g$. Set $g_{n}=T f_{n}$. Then, for each $n, g_{n} \in W$ by (4), and $-n \leqq g_{n} \leqq n$ by (8). Since $\|T f\| \leqq\|f\|$ for all $f$ (by (3)), and since $T g=g$ by (5), we have $\left\|g_{n}-g\right\|=\left\|T f_{n}-T g\right\|=\left\|T\left(f_{n}-g\right)\right\| \leqq\left\|f_{n}-g\right\|$, so that $\lim _{n \rightarrow \infty} g_{n}=g$. Since $g$ is arbitrary, $W$ is thus shown to be bounded.

According to (5) and (6), $W$ does contain every constant function. It remains therefore to show that $f \in W, g \in W$ implies $f \cdot g \in W$, provided that $f$ and $g$ are bounded. It will suffice to show that

$$
f \in W \text { implies } f^{2} \in W \text { provided that } f \text { is bounded. }
$$

For, if $f$ and $g$ are bounded functions in $W$ then $f+g$ is such a function also, and it will follow from (10) that $f^{2}, g^{2}$, and $(f+g)^{2}$ are in $W$; consequently $f \cdot g=(f+g)^{2} / 2-f^{2} / 2-g^{2} / 2$ is in $W$.

We proceed to establish (10). Choose and fix a bounded $f \in W$, and define $g=T f^{2}-f^{2}$. In view of (5), we have to show that $g=0$.

Consider the parabola $v=u^{2}$ in the $u v$-plane, and for any real $r$ let $v=a_{r} u+b_{r}$ be the tangent to the parabola at the point $u=r$, $v=r^{2}\left(a_{r}=2 r, b_{r}=-r^{2}\right)$. Let $R$ be a countable everywhere dense set (e.g. the rational points) of the real line. Then, for each $u,-\infty<u$ $<\infty, u^{2} \geqq a_{r} u+b_{r}$ for every $r$, and $u^{2}=\sup _{r \in R}\left\{a_{r} u+b_{r}\right\}$. 
We have $f^{2} \geqq a_{r} f+b_{r}$ for each fixed $r$. Hence, for each $r, T f^{2} \geqq T\left(a_{r} f\right.$ $\left.+b_{r}\right)=a_{r} f+b_{r}$, by (9), (1), (5), and (6). It follows that

$$
T f^{2} \geqq \sup _{r \in R}\left\{a_{r} f+b_{r}\right\}=f^{2} .
$$

Thus $g \geqq 0$. We observe next that, for any $h \in V$,

$$
\begin{aligned}
\int_{X} h d \mu & =(h, 1) \\
& =(h, T 1) \text { by }(6) \\
& =(T h, 1) \text { by }(3) \\
& =\int_{X} T h d \mu .
\end{aligned}
$$

Since $T g=T^{2} f^{2}-T f^{2}=T f^{2}-T f^{2}=0$ by (1) and (2), it follows from (11) that $\int_{X} g d \mu=0$, and hence $g=0$. This completes the proof that (i) $\rightarrow$ (ii).

Suppose now that (ii) holds. We shall show first that $S_{0}$ is a $\sigma$ algebra. Since $W$ contains the function $1, \chi_{A} \in W$ implies $\chi_{x-A}$ $=1-\chi_{A} \in W$; since $W$ is closed under pointwise multiplication of functions, $\chi_{A} \in W, \chi_{B} \in W$ implies $\chi_{A \cap_{B}}=\chi_{A} \cdot \chi_{B} \in W$; and since $W$ is a closed subset of $V$, if $f_{1}, f_{2}, \cdots$ is a sequence of characteristic functions in $W$ such that $f_{r} \cdot f_{s}=0$ for $r \neq s, g=\sum_{r} f_{r}$ is a characteristic function in $W$. By referring to the definition of $S_{0}$;-we see that the class $S_{0}$ is closed under complementation, under intersection, and under countable union of disjoint sets; $S_{0}$ is therefore a $\sigma$-algebra. We proceed to show that $S^{0} \subset S_{0}$; this will establish (iii), since $S_{0} \subset S^{0}$ in any case.

Let $f$ be a bounded function in $W$, and suppose that $\alpha \leqq f \leqq \beta$. Let $I$ denote the interval $[\alpha, \beta]$, and let $M$ be the class of Borel measurable sets of $I$. Define $\nu(C)=\mu\left(f^{-1}(C)\right)$ for $C \in M$. Then $\nu$ is a probability measure on $M$. It is well known (cf., e.g., [3] )that the set of polynomial functions on $I$ is dense in $\mathcal{L}_{2}(I, M, \nu)$. Let us consider a fixed $C \in M$. There exists a sequence $\left\{p_{n}\right\}$ of polynomials on $I$ such that $\lim _{n \rightarrow \infty} p_{n}=\chi_{C}$. Writing $A=f^{-1}(C)$, we have $\left\|p_{n}-\chi_{C}\right\|=\left\|p_{n}(f)-\chi_{A}\right\|$ for every $n$, so that $\lim _{n \rightarrow \infty} p_{n}(f)=\chi_{A}$. Since $W$ is algebraic, and $f$ is a bounded function in $W$, we have $p_{n}(f) \in W$ for each $n$; hence, since $W$ is closed, $\chi_{A} \in W$, that is to say, $A=f^{-1}(C) \in S_{0}$. Since $C$ is arbitrary, $f$ is $S_{0}$-measurable.

We have therefore shown that every bounded $f \in W$ is an $S_{0}$ measurable function. Since $W$ is bounded, it follows that every $f \in W$ is $S_{0}$-measurable. Consequently $S^{0} \subset S_{0}$, by the definition of $S^{0}$. This completes the proof that (ii) $\rightarrow$ (iii). 
Suppose next that $S_{0}$ is a $\sigma$-algebra, which is less than supposing that (iii) holds. Consider an $f \in \mathcal{L}_{2}\left(X, S_{0}, \mu\right)$. There exists a sequence $\left\{f_{n}\right\}$ such that each $f_{n}$ is of the form $\sum_{i=1}^{k} \alpha_{i} g_{i}$ where $g_{1}, g_{2}, \cdots, g_{k}$ are $S_{0}$-measurable characteristic functions, and also such that $\lim _{n \rightarrow \infty} f_{n}=f$. It follows from the definition of $S_{0}$ that $\left\{f_{n}\right\}$ is a sequence in $W$; hence $f \in W$, since $W$ is closed. Since $f$ is arbitrary, we have $\mathcal{L}_{2}\left(X, S_{0}, \mu\right) \subset W$. On the other hand, $f \in W$ implies (by the definition of $\left.S^{0}\right)$ that $f$ is $S^{0}$-measurable and therefore in $\mathcal{L}_{2}\left(X, S^{0}, \mu\right)$, so that $W \subset \mathcal{L}_{2}\left(X, S^{0}, \mu\right)$. Thus $\mathcal{L}_{2}\left(X, S_{0}, \mu\right) \subset W \subset \mathcal{L}_{2}\left(X, S^{0}, \mu\right)$, provided only that $S_{0}$ is a $\sigma$-algebra; if in fact $S_{0}=S^{0}$ the three subspaces must be identical. This proves, in particular, that (iii) $\rightarrow$ (iv).

Suppose, finally, that (iv) holds. Let $U$ be the conditional expectation operator relative to $S^{0}$, that is to say, for each $f$ in $\mathcal{L}_{1}(X$, $S, \mu)$ let $U f$ be the unique function in $\mathcal{L}_{1}\left(X, S^{0}, \mu\right)$ such that $\int_{A} f d \mu$ $=\int_{A} U f d \mu$ for all $A \in S^{0}$. The existence of such a function follows easily from the Radon-Nikodym theorem (cf., e.g., [4]). Consider an $f \in V$. Since $T f \in W$ by (4), the hypothesis (iv) implies that

$$
T f \in \mathcal{L}_{1}\left(X, S^{0}, \mu\right) \text {. }
$$

Also, for any $A \in S^{0}$ we have

$$
\begin{aligned}
\int_{A} f d \mu & =\left(f, \chi_{A}\right) \\
& =\left(f, T \chi_{A}\right) \text { by (iv) and (5) } \\
& =\left(T f, \chi_{A}\right) \text { by (3) } \\
& =\int_{A} T f d \mu .
\end{aligned}
$$

It follows hence by the uniqueness of conditional expectation that $T f$ differs from $U f$ on a set of $\mu$-measure zero. Since $f$ is arbitrary, we conclude that $T=U$ on $V$. (It follows incidentally that $f \in V$ implies $U f \in V,\|U f\| \leqq\|f\|)$. As is well known, $U$ is constant-preserving and positive, so that (i) holds. This completes the proof of the theorem.

3. Applications to a finite-dimensional function space. By way of an example, let $X=\{1,2, \cdots, n\}, S=$ the class of all sets of $X$, and $\mu=$ the uniform distribution on $X$, that is to say, $\mu(\{x\})=1 / n$ for $x=1,2, \cdots, n$. In this case $V$ is the real vector space of all realvalued functions on $X$, every $f \in V$ is bounded, and every linear manifold in $V$ is closed.

Let $W$ be a linear manifold in $V$. By definition, $W$ is algebraic if it contains every constant function, and if $W$ is closed under pointwise 
multiplication of functions. It follows from Corollary 1 that a $k$ dimensional linear manifold $W$ is algebraic if and only if there exists a partition of $X$ into $k$ mutually exclusive nonempty sets, $X=\mathrm{U}_{r-1}^{k} X_{r}$ say, such that $W$ is the set of all functions of the form $f(x)=\alpha_{r}$ for $x \in X_{r}(r=1,2, \cdots, k)$. The verification is omitted.

For each $i=1,2, \cdots, n$ let $f_{i}(x)=1$ for $x=i$ and $f_{i}(x)=0$ for $x \neq i$. Then $\left\{f_{1}, f_{2}, \cdots, f_{n}\right\}$ is a basis in $V$. Let $\left\{t_{i j}\right\}$ be an $n \times n$ symmetric probability matrix (i.e. $t_{i j}=t_{j i}, t_{i j} \geqq 0, \sum_{j=1}^{n} t_{i j}=1$ ), and for $f^{\prime}=\sum_{i=1}^{n} \alpha_{i} f_{i}$ let $T f=\sum_{i=1}^{n} \alpha_{i} T f_{i}$, where $T f_{i}=\sum_{j=1}^{n} t_{i j} f_{j}$. Then the transformation $T$ is linear, self-adjoint, constant-preserving, and positive.

Clearly, $T$ is idempotent if and only if the matrix $\left\{t_{i j}\right\}$ is idempotent (i.e. $\sum_{r=1}^{n} t_{i r} t_{r j}=t_{i j}$ ). Moreover, it follows easily from the definition of a conditional probability that $T$ is a conditional expectation, with a $k$-dimensional subspace as its range, if and only if there exists a partition of $n$ into $k$ positive integers, $n=n_{1}+n_{2}+\ldots$ $+n_{k}$ say, such that (except for a permutation of rows and columns) $\left\{t_{i j}\right\}$ is the matrix

$$
M_{k}\left(n_{1}, n_{2}, \cdots, n_{k}\right)=\left(\begin{array}{c:ccc}
N_{1} & & & 0 \\
& N_{2} & & \\
& & \ddots & \\
0 & & N_{k}
\end{array}\right) .
$$

where $N_{r}$ is the $n_{r} \times n_{r}$ matrix with each element equal to $1 / n_{r}$.

It now follows from Corollary 2 that a symmetric $n \times n$ probability matrix of rank $k$ is idempotent if and only if there exists a partition of $n$ into $k$ positive integers $n_{1}, n_{2}, \cdots, n_{k}$ such that the matrix is a permutation of the rows and columns of $M_{k}\left(n_{1}, n_{2}, \cdots, n_{k}\right)$.

\section{REFERENCES}

1. Shu-Teh Chen Moy, Characterizations of conditional expectation as a transformation on function spaces, Pacific Journal of Mathematics vol. 4 (1954) p. 47.

2. P. R. Halmos, Introduction to Hilbert space and the theory of spectral multiplicity, New York, Chelsea, 1951, pp. 1-48.

3. G. Szegö, Orthogonal polynomials, Amer. Math. Soc. Colloquium Publications, vol. 23, 1939, pp. 10-11.

4. J. L. Doob, Stochastic processes, New York, Wiley, 1953, pp. 15-18.

5. P. R. Halmos, Measure theory, New York, Van Nostrand, 1951.

6. G. Birkhoff, Moyennes des fonctions bornées, Congrés d'Algèbre et Théorie des Nombres, Paris, 1949, pp. 143-156.

The University of Chicago 